\title{
CHARACTERIZATION OF DISKCYCLIC OPERATORS
}

\author{
Abdallah M. Abu Jahel,1* \\ As'ad Y. As'ad,2 \\ 1Department of Mathematics, Faculty of Sciences, \\ Islamic University of Gaza, Gaza Strip, Palestine. \\ * Corresponding author \\ e-mail address: aabujahel@iugaza.edu.ps
}

Abstract:

In this article we discuss an interesting idea related to characterization of diskcyclicity phenomena. We use the Diskcyclic Criterion to characterize the subsets $\Gamma$ of $\mathbb{C}$ for which the notion of $\Gamma$-supercyclicity coincides with the notion of diskcyclicity. In particular, we give a complete description of the subset $\Gamma$. The necessity and sufficiency conditions on the subset $\Gamma$ were presented. Some examples were exhibited to illustrate the proofs and the relationship among different classes of the operators.
Keywords:

$\Gamma$-supercyclicity, diskcyclic operators, hypercyclic scalar set, supercyclic operators 


\section{Introduction}

Let $\mathcal{H}$ be a separable complex Hilbert space, $X$ be a separable complex Banach space and let $\mathcal{B}(X)$ be the Banach algebra of all bounded linear operators on $X$. We will refer to the closed unit disk in the complex plane by $\mathbb{D}$, i.e. $\mathbb{D}:=\{\gamma \in \mathbb{C} ;|\gamma| \leq 1\}$, for other disks in the complex plane with radius $r>0$ by $\mathbb{D}_{r}$, i.e. $\mathbb{D}_{r}:=\{\gamma \in \mathbb{C} ;|\gamma| \leq r\}$, the open unit disk by $\mathbb{U}:=\{\gamma \in \mathbb{C} ;|\gamma|<1\}$ and the unit circle by $\mathbb{T}:=\{\gamma \in \mathbb{C} ;|\gamma|=1\}$. For $T$ in $\mathcal{B}(X), x$ in $X$, and $\Gamma \backslash\{0\}$ a non-empty subset of the complex plane $\mathbb{C}$, we denote $\operatorname{Orb}(\Gamma x, T)=\left\{\gamma T^{n} x: \gamma \in \Gamma, n \geq 0\right\}$. We say that $x$ is a $\Gamma$-supercyclic vector for $T$ if $\operatorname{Orb}(\Gamma x, T)$ is dense in $X$ and $T$ will be called $\Gamma$-supercyclic if it has a $\Gamma$-supercyclic vector. Recall that an element $x$ in $X$ is a hypercyclic vector for $T$ if the set $\left\{T^{n} x: n \geq 0\right\}$ is dense in $X$, a supercyclic vector if the set $\left\{\gamma T^{n} x: \gamma \in \mathbb{C}, n \geq 0\right\}$ is dense in $X$ and a diskcyclic vector if the set $\left\{\gamma T^{n} x: \gamma \in \mathbb{D}, n \geq 0\right\}$ is dense in $X$. So, one can verify that a $\Gamma$-supercyclic operator is hypercyclic whenever $\Gamma=\{1\}$, supercyclic if $\Gamma=\mathbb{C}$ and diskcyclic if the set $\Gamma=\mathbb{D}$.

In this article we will refer to diskcyclic operator by $\mathbb{D}$-supercyclic and the other types by the same way corresponding to their subset $\Gamma \subset \mathbb{C}, \Gamma^{\circ}$ is the interior of the set $\Gamma$. Also when we say $\mathbb{N}$ we mean the set $\{0,1,2, \ldots\}$.

For $x \in \ell^{2}(\mathbb{N})$ the unilateral forward shift is the operator $F$ defined by $F x=F\left(x_{0}, x_{1}, x_{2}, \ldots\right)=$ $\left(0, x_{0}, x_{1}, x_{2}, \ldots\right)$ and in terms of the canonical basis $\left\{e_{n}\right\}_{n \geq 0}$ in $\ell^{2}(\mathbb{N})$ is $F e_{n}=e_{n+1}$. The corresponding operator in $\ell^{2}(\mathbb{Z})$ is the bilateral forward shift operator defined by $F\left(\ldots, x_{-1}, x_{0}, x_{1}, \ldots\right)=$ $\left(\ldots, x_{-2}, x_{-1}, x_{0}, \ldots\right)$ and in terms of the canonical basis $\left\{e_{n}\right\}_{n \in \mathbb{Z}}$ in $\ell^{2}(\mathbb{Z})$ is $F e_{n}=e_{n+1}$. On the other hand the unilateral backward shift operator $B$ on $\ell^{2}(\mathbb{N})$ is defined by, $B x=B\left(x_{0}, x_{1}, x_{2}, \ldots\right)=\left(x_{1}, x_{2}, x_{3}, \ldots\right)$ and in terms of the canonical basis in $\ell^{2}(\mathbb{N})$ is $F e_{n}=$ $e_{n-1}$, where $e_{0}=(1,0,0, \ldots)$. The corresponding operator on $\ell^{2}(\mathbb{Z})$ is the bilateral backward shift operator defined by $B\left(\ldots, x_{-1}, x_{0}, x_{1}, \ldots\right)=\left(\ldots, x_{0}, x_{1}, x_{2}, \ldots\right)$.

Let $X=\ell^{2}(\mathbb{Z})$ and $\left\{\alpha_{n}\right\}$ be a bounded sequence of positive numbers for all $n \in \mathbb{Z}$. The operator $F_{\alpha} \in \mathcal{B}(X)$ is called forward weighted shift operator if $F_{\alpha}\left(\ldots, x_{-1}, x_{0}, x_{1}, \ldots\right)=$ $\left(\ldots, \alpha_{-2} x_{-2}, \alpha_{-1} x_{-1}, \alpha_{0} x_{0}, \ldots\right)$. However, the operator $B_{\alpha} \in \mathcal{B}(X)$ is called backward weighted shift operator if $B_{\alpha}\left(\ldots, x_{-1}, x_{0}, x_{1}, \ldots\right)=\left(\ldots, \alpha_{-1} x_{0}, \alpha_{0} x_{1}, \alpha_{1} x_{2}, \ldots\right)$.

Let $\left\{e_{n}\right\}_{n \in \mathbb{Z}}$ is the canonical basis in $\ell^{2}(\mathbb{Z})$, note that for each $n \in \mathbb{Z}$ and $k \in \mathbb{N}$,

$$
F_{\alpha}^{k} e_{n}=\left(\prod_{n}^{n+k-1} \alpha_{j}\right) e_{n+k} \text { has inverse operator as } B_{\frac{1}{\alpha}} e_{n}=\left(\prod_{n-1}^{n-k} \frac{1}{\alpha_{j}}\right) e_{n-k} .
$$

The class of weighted shifts is suitable for finding examples of different types of operators. So we will depend on this family to construct our examples in this article.

The definition of hypercyclicity was already studied by G.D. Birkhoff [2] in 1922. Also he proved the first characterization of hypercyclic operators which is a direct application of the Baire category theorem. Now it is often referred to as Birkhoff's transitivity Theorem [2]. In 1929 G.D. Birkhoff [3] gave a historical example of hypercyclic operator. Later G.R. MacLane [13] found the same phenomenon for the differentiation operator. In the beginning of eighteenth linear operators theory is rapidly evolving in a branch of functional analysis, which was probably born in 1982 with the Toronto 
Ph.D. thesis of C. Kitai [8]. After that it has become rather popular by the efforts of many mathematicians. In the early seventies, Hilden and Wallen [10] introduced the definition of supercyclicity. The notation of diskcyclicity was already studied by Zeana [11].

One of the most important results from [11] was the Diskcyclicity Criterion.

Theorem (Diskcyclicity Criterion). [11] Let $T \in \mathcal{B}(X)$. We assume that there exist two dense subsets $X_{0}, Y_{0} \subset X$, an increasing sequence $\left(n_{k}\right)_{k \in \mathbb{N}} \subset \mathbb{N}$, and maps $S_{n_{k}}: Y_{0} \rightarrow X$ such that for any $x \in X_{0}$ and any $y \in Y_{0}$ the following holds:

1. $T^{n_{k}} x \rightarrow 0$ as $k \rightarrow \infty$;

2. $S_{n_{k}} y \rightarrow 0$ as $k \rightarrow \infty$;

3. $T^{n_{k}} S_{n_{k}} y \rightarrow y$ as $k \rightarrow \infty$.

Then $T$ is diskcyclic.

Later, in 2015 Bamerni, Kilicman and Noorani [1] presented a another equivalent version of the Diskcyclicity Criterion.

Theorem (Second Diskcyclicity Criterion). [1] Let $T \in \mathcal{B}(X)$. If there exists an increasing sequence of integers $\left(n_{k}\right)_{k \in \mathbb{N}}$ and two dense sets $D_{1}, D_{2} \subset X$ such that

1. for each $y \in D_{2}$, there is a sequence $\left\{x_{k}\right\}$ in $X$ such that $x_{k} \rightarrow 0$ and $T^{n_{k}} x_{k} \rightarrow y$,

2. $\left\|T^{n_{k}} x\right\|\left\|x_{k}\right\| \rightarrow 0$ for all $x \in D_{1}$.

Then $T$ is diskcyclic.

Zeana [11] asked "Is every $\mathbb{T}$-supercyclic operator a hypercyclic operator?". León and Müller in [12] proved that $T$ is a hypercyclic operator if and only if $T$ is a $\mathbb{T}$-supercyclic operator in the following Theorem.

Theorem (León-Müller Theorem).

Let $T \in \mathcal{B}(X)$. Then $x \in X$ is a hypercyclic for $T$ if and only if $x$ is a $\mathbb{T}$-supercyclic for $T$.

It is worth to mention that, as a result of this theorem, León and Müller proved that for any complex number $\lambda$ with modulus $1, T$ is hypercyclic if and only if $\lambda T$ is hypercyclic with the same hypercyclic vectors. These results answering a question posed by Herrero [9].

It is important to highlight that when we enlarge the orbit or the scaled orbit of a such vector we may lost the equivalency of the orbit and the enlarge orbit. For example if the vector $x$ is a hypercyclic vector for $T$ then $\operatorname{Orb}(x, T)$ is dense in $X$. Now, if we enlarge $\operatorname{Orb}(x, T)$ by $\operatorname{Orb}(\mathbb{D} x, T)$ we can get a $\mathbb{D}$-supercyclic vector for $T$ which is not a hypercyclic vector for $T$. In other word, the two sets $\operatorname{Orb}(x, T)$ and $\operatorname{Orb}(\mathbb{D} x, T)$ are not equivalent. To show some examples we recommend to see [1].

One of the general problems in this field is "what is the big set $\Gamma \subset \mathbb{C}$ which is still ensure diskcyclic phenomena. 
In 2002 Feldman [7] investigated this problem in a different approach. He characterized the hypercyclicity by research to the small sets that has orbit under $T$ to still ensure the hypercyclicity. He proved that the operator is hypercyclic if and only if there exist $d>0$ and a vector $x \in \mathcal{H}$ having a $d$-dense orbit.

In 2016, Charpentier, Ernst and Menet in [5] presented a wonderful characterization of the subset $\Gamma \subset \mathbb{C}$ that still ensure hypercyclicity. They say that the subset $\Gamma$ must be bounded and bounded away from zero.

Later, in 2020 Charpentier and Ernst in [4] gave a complete characterization of the finite dimensional subsets $C$ of any separable Hilbert space for which the notion of $C$-hypercyclicity coincides with the notion of hypercyclicity, where an operator $T$ on a topological vector space $X$ is said to be $C$-hypercyclic if the set $T^{n} x: n \geq 0, x \in C$ is dense in $X$. They gave a partial description for non necessarily finite dimensional subsets.

Theorem. [5] A non-empty subset $\Gamma$ of $\mathbb{C}$ is a hypercyclic scalar set if and only if $\Gamma \backslash\{0\}$ is non-empty, bounded and bounded away from 0.

So, it is natural to ask: Can these results be extended to the diskcyclic operators or not?

In this article we ask what is the biggest subset $\Gamma \subset \mathbb{C}$ that still equivalent with $\mathbb{D}$.

Question 1. Is it possible to characterize the sets $\Gamma \subset \mathbb{C}$ such that $T$ is diskcyclic if and only if $\operatorname{Orb}(\Gamma x, T)$ is dense?

The main purpose of this article is to discuss Question 1. So in order to deal with Question 1 we will present some properties of subsets of $\mathbb{C}$. In fact, we will positively answering Question 1 and give a necessary and sufficient conditions on that sets in $\mathbb{C}$ which still ensure diskcyclicity.

The article is organized as follows. In section 2 we give the inspired result that lead us to ask Question 1 and search about a suitable answer. In section 3 we provide the necessity part of Theorem A and in section 4 we present a sufficiency part of Theorem A. Finally, we give a conclusion in the last section.

\section{Main Results}

In our work in this field we notice that, for any fixed positive real number $r$, and $\Gamma=\mathbb{D}_{r}$, the operator $T$ is $\Gamma$-supercyclic if and only if $T$ is $\mathbb{D}$-supercyclic.

Theorem 2.1. Let $r$ be a fixed positive real number. Then $T \in \mathcal{B}(X)$ is a $\mathbb{D}$-supercyclic operator if and only if $T$ is a $\mathbb{D}_{r}$-supercyclic operator.

\section{Proof.}

Suppose that $T$ is a $\mathbb{D}_{r}$-supercyclic operator with $x$ as a $\mathbb{D}_{r}$-supercyclic vector. Let $\varepsilon>0$ be given. For any $y \in X$, we have $\alpha \in \mathbb{D}_{r}$ and $n \in \mathbb{N}$ such that $\left\|\alpha T^{n} x-r y\right\|<r \varepsilon$, which implies $r\left\|\frac{\alpha}{r} T^{n} x-y\right\|<$ $r \varepsilon$. Thus, $\left\|\frac{\alpha}{r} T^{n} x-y\right\|<\varepsilon$. Clearly, $\left|\frac{\alpha}{r}\right| \leq 1$. This shows that, for any $\varepsilon>0$ and a non-zero $y \in X$, we have an element in $\left\{\lambda T^{n} x: n \in \mathbb{N}, \lambda \in \mathbb{D}\right\}$ such that $\left\|\lambda T^{n} x-y\right\|<\varepsilon$. In other word, the 
set $\left\{\lambda T^{n} x: n \in \mathbb{N}, \lambda \in \mathbb{D}\right\}$ is dense in $X$. Hence, $T$ is a $\mathbb{D}$-supercyclic operator with the same $\mathbb{D}$-supercyclic vector $x$. Conversely, suppose that $T$ is a $\mathbb{D}$-supercyclic operator with $\mathbb{D}$-supercyclic vector $x$. Let $\varepsilon>0$ be given. For any $y \in X$, we have $\lambda \in \mathbb{D}$ and $n_{1} \in \mathbb{N}$ such that $\left\|\lambda T^{n_{1}} x-\frac{1}{r} y\right\|<\frac{\varepsilon}{r}$. So, $\frac{1}{r}\left\|r \lambda T^{n_{1}} x-y\right\|<\frac{\varepsilon}{r}$. Then, $\left\|r \lambda T^{n_{1}} x-y\right\|<\varepsilon$. Clearly, $|r \lambda| \leq r$. This shows that, for any $\varepsilon>0$ and a non-zero $y \in X$, we have an element in $\left\{\lambda T^{n} x: n \in \mathbb{N}, \lambda \in \mathbb{D}_{r}\right\}$ such that $\left\|\lambda T^{n} x-y\right\|<\varepsilon$. Hence, $T$ is a $\mathbb{D}_{r}$-supercyclic operator with the same $\mathbb{D}_{r}$-supercyclic vector $x$.

The results from [5] and Theorem 2.1 inspire us to reach the characterization of the big sets in $\mathbb{C}$ that still ensure the diskcyclicity. In order to present this characterization we must investigate some properties that subsets in $\mathbb{C}$ can enjoy it or not.

Definition 2.2. Let $\Gamma$ be a subset of $\mathbb{C}$. $\Gamma$ is said to be a diskcyclic scalar set if the following holds true: For every complex Banach space $X$, every $T \in \mathcal{B}(X)$ and every $x \in X$

$x$ is a $\Gamma$-supercyclic vector for $T$ if and only if $x$ is a diskcyclic vector for $T$.

We introduce a complete answer to the Question 1 by the following theorem.

Theorem A. A non-empty subset $\Gamma$ of $\mathbb{C}$ is a diskcyclic scalar set if and only if $\Gamma \backslash\{0\}$ is non-empty, bounded and $0 \in \Gamma^{\circ}$.

\section{NECESSARY PART OF THEOREM A}

In order to prove Theorem A we begin by the first direction, that is, if $\Gamma$ is a diskcyclic scalar set then $\Gamma \backslash\{0\}$ is non-empty, bounded and $0 \in \Gamma^{\circ}$. It is worth mentioning that, if $\Gamma$ is a diskcyclic scalar set then obviously $\Gamma \backslash\{0\}$ must be non-empty. So it remains to show that $\Gamma$ is bounded and $0 \in \Gamma^{\circ}$. We will prove this direction by contraposition. By definition (2.2) it suffices to exhibit three examples. The first example is a $\Gamma$-supercyclic operator which is not a $\mathbb{D}$-supercyclic operator whenever $\Gamma$ is an unbounded subset of $\mathbb{C}$. The second example is a $\mathbb{D}$-supercyclic operator which is not a $\Gamma$-supercyclic operator whenever 0 is in the boundary of the set $\Gamma$. The last example is a $\mathbb{D}$-supercyclic operator which is not a $\Gamma$-supercyclic whenever $0 \notin \bar{\Gamma}$.

Example 3.1. Let $\Gamma$ be an unbounded subset of $\mathbb{C}$. Then the backward shift operator $B$ on $\ell^{2}(\mathbb{N})$ is $\Gamma$-supercyclic but not $\mathbb{D}$-supercyclic.

Proof. It is well known that, for any non-zero vector $x$, the orbit $\operatorname{Or} b(x, B)=\left\{x, B x, B^{2} x, B^{3} x, \ldots\right\}$ is bounded by $\|x\|$. So, there is no diskcyclic vector for $B$ and hence $B$ is not a diskcyclic operator. We are going to show that $B$ is a $\Gamma$-supercyclic operator. So we want to find a $\Gamma$-supercyclic vector for $B$. Let $c_{00}(\mathbb{N})$ be the subspace of finite support vectors of $\ell^{2}(\mathbb{N})$ which is defined by $c_{00}(\mathbb{N})=$ $\left\{y_{k}(j) \in \ell^{2}(\mathbb{N}): \exists j_{0} \in \mathbb{N}\right.$ such that $\left.y_{k}(j)=0, \forall j>j_{0}\right\}$. Recall that $c_{00}(\mathbb{N})$ is a countable dense subspace of $\ell^{2}(\mathbb{N})$. For every $k \in \mathbb{N}$ take $y_{k} \in c_{00}(\mathbb{N})$ and define $d\left(y_{k}\right)=\max \left\{j \geq 0: y_{k}(j) \neq 0\right\}$. For any vector $y \in \ell^{2}(\mathbb{N})$ we can find a sequence of vectors $\left(y_{k}\right)_{k \in \mathbb{N}}$ in $c_{00}(\mathbb{N})$ such that $\left\|y_{k}-y\right\| \rightarrow 0$ as $k \rightarrow \infty$. Since $\Gamma$ is an unbounded set of $\mathbb{C}$. Then we can construct an unbounded sequence 
$\left(\gamma_{k}\right)_{k \in \mathbb{N}} \subset \Gamma$ such that for every $y_{k}$, there is $\gamma_{k}$ such that $\left\|\frac{1}{\gamma_{k}} y_{k}\right\|<\frac{1}{2^{k}}$ and $\frac{\left|\gamma_{i}\right|}{\left|\gamma_{k}\right|}\left\|y_{k}\right\|<\frac{1}{2^{k}}$ for every $i<k$ (which is possible because for any $k \in \mathbb{N}$ we can find a such $\gamma_{k}$ that has suitable big modulus such that $\left.\left\|\frac{1}{\gamma_{k}} y\right\|=\frac{1}{\left|\gamma_{k}\right|}\|y\|<\frac{1}{2^{k}}\right)$. Finally, we are going to construct a sequence of integers $\left(m_{k}\right)_{k \in \mathbb{N}}$ as $m_{0}=0, m_{1}>m_{0}+d\left(y_{0}\right), m_{2}>m_{1}+d\left(y_{1}\right) \ldots$ In general, $m_{k}>m_{k-1}+d\left(y_{k-1}\right), k=1,2,3, \ldots$. Now, take the vector $x=\sum_{i=0}^{\infty} \frac{1}{\gamma_{i}} F^{m_{i}} y_{i}$, where $F$ is the forward shift operator on $\ell^{2}(\mathbb{N})$. Recall that $\left\|F^{m_{i}} y_{i}\right\|=\left\|y_{i}\right\|$. Hence, $\|x\|=\left\|\sum_{i=0}^{\infty} \frac{1}{\gamma_{i}} F^{m_{i}} y_{i}\right\| \leq \sum_{i=0}^{\infty} \frac{1}{\left|\gamma_{i}\right|}\left\|F^{m_{i}} y_{i}\right\|=\sum_{i=0}^{\infty}\left\|\frac{1}{\gamma_{i}} y_{i}\right\|<\sum_{i=0}^{\infty} \frac{1}{2^{i}}=$ 2. Hence, $x \in \ell^{2}(\mathbb{N})$. We claim that $x$ is a $\Gamma$-supercyclic vector for $B$. Let $k \in \mathbb{N}$. We have

$$
\begin{aligned}
\left\|\gamma_{k} B^{m_{k}} x-y_{k}\right\| & =\left\|\gamma_{k} B^{m_{k}} \sum_{i=0}^{\infty} \frac{1}{\gamma_{i}} F^{m_{i}} y_{i}-y_{k}\right\|=\left\|\sum_{i=0}^{\infty} \frac{\gamma_{k}}{\gamma_{i}} B^{m_{k}} F^{m_{i}} y_{i}-y_{k}\right\| \\
& =\left\|\sum_{i<k} \frac{\gamma_{k}}{\gamma_{i}} B^{m_{k}} F^{m_{i}} y_{i}+\frac{\gamma_{k}}{\gamma_{k}} B^{m_{k}} F^{m_{k}} y_{k}-y_{k}+\sum_{i>k} \frac{\gamma_{k}}{\gamma_{i}} B^{m_{k}} F^{m_{i}} y_{i}\right\| \\
& \leq \sum_{i<k}\left\|\frac{\gamma_{k}}{\gamma_{i}} B^{m_{k}} F^{m_{i}} y_{i}\right\|+\left\|\frac{\gamma_{k}}{\gamma_{k}} B^{m_{k}} F^{m_{k}} y_{k}-y_{k}\right\|+\sum_{i>k}\left\|\frac{\gamma_{k}}{\gamma_{i}} B^{m_{k}} F^{m_{i}} y_{i}\right\| \\
& \left.\leq 0+0+\sum_{i>k} \frac{\left|\gamma_{k}\right|}{\left|\gamma_{i}\right|}\left\|y_{i}\right\| \text { (the first zero because } m_{k}>m_{i}+d\left(y_{i}\right)\right) \\
& \leq \sum_{i>k} \frac{1}{2^{k}}=\frac{1}{2^{k}} \stackrel{\text { as } k \rightarrow \infty}{\longrightarrow} \quad 0 .
\end{aligned}
$$

For any $y \in \ell^{2}(\mathbb{N})$, there is $\left(y_{k}\right)_{k \in \mathbb{N}} \in c_{00}(\mathbb{N})$ such that $\left\|y_{k}-y\right\| \stackrel{\text { as } k \rightarrow \infty}{\longrightarrow} 0$. So we have,

$$
\left\|\gamma_{k} B^{m_{k}} x-y\right\| \leq\left\|\gamma_{k} B^{m_{k}} x-y_{k}\right\|+\left\|y_{k}-y\right\| \stackrel{\text { as } k \rightarrow \infty}{\longrightarrow} 0+0=0
$$

where, $\gamma_{k} \in \Gamma$. Therefore $x$ is a $\Gamma$-supercyclic vector for $B$.

The zero element plays an important role in the subset $\Gamma$. It is worth mentioning that if $\Gamma$ is not a closed subset of $\mathbb{C}$ and $0 \notin \Gamma$, then 0 may be in the boundary of $\Gamma$ or $0 \notin \bar{\Gamma}$. Then we have two cases. Now we consider the case when $0 \notin \bar{\Gamma}$.

Example 3.2. Let $\Gamma$ be a subset of $\mathbb{C}$ and $0 \notin \bar{\Gamma}$. Let $B_{\omega}: \ell^{2}(\mathbb{Z}) \longrightarrow \ell^{2}(\mathbb{Z})$ be the backward weighted shift operator with weight sequence

$$
\omega_{n}=\left\{\begin{array}{l}
4 \text { if } n>0 \\
1 \text { otherwise. }
\end{array}\right.
$$

Then $B_{\omega}$ is $\mathbb{D}$-supercyclic but is not $\Gamma$-supercyclic.

Proof. Let $\Gamma$ be a subset of $\mathbb{C}$ such that $0 \notin \bar{\Gamma}$. It is not so hard to notice that the scaled orbit of any non-zero vector $x \in \ell^{2}(\mathbb{Z}), \operatorname{Orb}\left(x, B_{\omega}\right)$ is bounded below by $\|x\|$ and $\operatorname{Orb}\left(\Gamma x, B_{\omega}\right)$ is 
bounded away from 0 because $0 \notin \bar{\Gamma}$. So we can conclude that there are no $\Gamma$-supercyclic vectors for $B_{\omega}$ and hence $B_{\omega}$ is not $\Gamma$-supercyclic. On the other hand we claim that the operator $B_{\omega}$ is $\mathbb{D}$-supercyclic. So we want to find a $\mathbb{D}$-supercyclic vector for $B_{\omega}$. Let $c_{00}(\mathbb{Z})=\left\{y_{k} \in \ell^{2}(\mathbb{Z})\right.$ : $y_{k}$ has finitely many non-zero coordinates $\}$ be the countable dense subspace in $\ell^{2}(\mathbb{Z})$. For any $k \in \mathbb{N}$ take $y_{k} \in c_{00}(\mathbb{Z})$ and define $d\left(y_{k}\right)=\max \left\{j \geq 0: y_{k}(j) \neq 0\right\}$. Define the forward weighted shift operator $F_{\nu}: \ell^{2}(\mathbb{Z}) \longrightarrow \ell^{2}(\mathbb{Z})$ with weight sequence

$$
\nu_{n}= \begin{cases}\frac{1}{4} & \text { if } n \geq 0 \\ 1 & \text { otherwise. }\end{cases}
$$

Clearly, $F_{\nu}$ is the inverse operator of $B_{\omega}$. For every $k \in \mathbb{N}$ we can construct a sequence of integers $\left(m_{k}\right)_{k \in \mathbb{N}}$ and a sequence $\left(\gamma_{k}\right)_{k \in \mathbb{N}} \subset \mathbb{D}$ satisfying the three following statements:

1. $\left\|\frac{1}{\gamma_{k}} F_{\frac{1}{\omega}}^{m_{k}} y_{k}\right\|<\frac{1}{2^{k}}$,

2. $\frac{\left|\gamma_{i}\right|}{\left|\gamma_{k}\right|}\left\|B_{\omega}^{m_{i}} F_{\frac{1}{\omega}}^{m_{k}} y_{k}\right\|<\frac{1}{2^{k}}$, when $i>k$.

3. $\frac{\left|\gamma_{i}\right|}{\left|\gamma_{k}\right|}\left\|B_{\omega}^{m_{i}} F_{\frac{1}{\omega}}^{m_{k}} y_{k}\right\|<\frac{1}{2^{k}}$, when $i<k$,

In order to construct the above sequences, one can choose an arbitrary $\gamma_{0} \in \mathbb{D}$ and a suitable $m_{0}$ satisfying (1). After that, there are many way to choose $\gamma_{1}, \gamma_{2}, \ldots$ and $m_{1}, m_{2}, \ldots$ Here we introduce this procedure

1. Consider $\gamma_{0}$ and $m_{0}$ were chosen.

2. Take $m_{i}>m_{i-1}+d\left(y_{i-1}\right)$.

3. compute $a_{i}:=\frac{1}{\left|\gamma_{i-1}\right|}\left\|B_{\omega}^{m_{i}} F_{\frac{1}{\omega}}^{m_{i-1}} y_{i-1}\right\|$.

4. Take $\gamma_{i} \in \mathbb{D}$ such that $\left|\gamma_{i}\right|<\frac{1}{2^{i} a_{i}}$.

5. Check if $\left\|\frac{1}{\gamma_{i}} F_{\frac{1}{\omega}}^{m_{i}} y_{i}\right\|<\frac{1}{2^{i}}$ and $\frac{\left|\gamma_{i-1}\right|}{\left|\gamma_{i}\right|}\left\|B_{\omega}^{m_{i-1}} F_{\frac{1}{\omega}}^{m_{i}} y_{i}\right\|<\frac{1}{2^{i}}$.

a. if 5 is not true, return to 2 and increases $m_{i}$.

b. if 5 is true, put $i=i+1$, return to 2 and continue the procedure.

Now, we can say that for every $y_{k} \in c_{00}(\mathbb{Z})$ we have a corresponding $\gamma_{k} \in \mathbb{D}$ and $m_{k}$.

After the construction of $\left(\gamma_{k}\right)_{k \in \mathbb{N}}$ and $m_{k}$, take the vector $x=\sum_{k=0}^{\infty} \frac{1}{\gamma_{k}} F_{\frac{1}{\omega}}^{m_{k}} y_{k}$. Clearly, $x \in \ell^{2}(\mathbb{Z})$. We claim that $x$ is a $\mathbb{D}$-supercyclic vector for $B_{\omega}$. Let $k \in \mathbb{N}, y_{k} \in c_{00}(\mathbb{Z})$, we have the corresponding 
$\gamma_{k} \in \mathbb{D}$ and an integer $m_{k}$ such that,

$$
\begin{aligned}
\left\|\gamma_{k} B_{\omega}^{m_{k}} x-y_{k}\right\|= & \left\|\gamma_{k} B_{\omega}^{m_{k}}\left(\sum_{j=0}^{\infty} \frac{1}{\gamma_{j}} F_{\frac{1}{\omega}}^{m_{j}} y_{j}\right)-y_{k}\right\| \\
& =\left\|\sum_{j=0}^{\infty} \frac{\gamma_{k}}{\gamma_{j}} B_{\omega}^{m_{k}} F_{\frac{1}{\omega}}^{m_{j}} y_{j}-y_{k}\right\| \\
& =\left\|\sum_{j<k} \frac{\gamma_{k}}{\gamma_{j}} B_{\omega}^{m_{k}} F_{\frac{1}{\omega}}^{m_{j}} y_{j}+\frac{\gamma_{k}}{\gamma_{k}} B_{\omega}^{m_{k}} F_{\frac{1}{\omega}}^{m_{k}} y_{k}-y_{k}+\sum_{j>k} \frac{\gamma_{k}}{\gamma_{j}} B_{\omega}^{m_{k}} F_{\frac{1}{\omega}}^{m_{j}} y_{j}\right\| \\
& \leq\left\|\sum_{j<k} \frac{\gamma_{k}}{\gamma_{j}} B_{\omega}^{m_{k}} F_{\frac{1}{\omega}}^{m_{j}} y_{j}\right\|+\left\|\frac{\gamma_{k}}{\gamma_{k}} B_{\omega}^{m_{k}} F_{\frac{1}{\omega}}^{m_{k}} y_{k}-y_{k}\right\|+\left\|\sum_{j>k} \frac{\gamma_{k}}{\gamma_{j}} B_{\omega}^{m_{k}} F_{\frac{1}{\omega}}^{m_{j}} y_{j}\right\| \\
& \leq \sum_{j<k}\left\|\frac{\gamma_{k}}{\gamma_{j}} B_{\omega}^{m_{k}} F_{\frac{1}{\omega}}^{m_{j}} y_{j}\right\|+\left\|y_{k}-y_{k}\right\|+\sum_{j>k}\left\|\frac{\gamma_{k}}{\gamma_{j}} B_{\omega}^{m_{k}} F_{\frac{1}{\omega}}^{m_{j}} y_{j}\right\| \\
& <\sum_{j<k} \frac{1}{2^{k}}+0+\sum_{j>k} \frac{1}{2^{j}}=\frac{k}{2^{k}}+\frac{1}{2^{k+2}}<\frac{k}{2^{k}}+\frac{1}{2^{k}}=\frac{k+1}{2^{k}} \stackrel{\text { as } k \rightarrow \infty}{\longrightarrow} 0 .
\end{aligned}
$$

Now, for any $y \in \ell^{2}(\mathbb{Z})$, there is $\left(y_{k}\right)_{k \in \mathbb{N}} \subset c_{00}(\mathbb{Z})$ such that, $\left\|y_{k}-y\right\| \stackrel{\text { as } k \rightarrow \infty}{\longrightarrow} 0$. So we have,

$$
\left\|\gamma_{k} B_{\omega}^{m_{k}} x-y\right\| \leq\left\|\gamma_{k} B_{\omega}^{m_{k}} x-y_{k}\right\|+\left\|y_{k}-y\right\| \quad \stackrel{\text { as } k \rightarrow \infty}{\longrightarrow} \quad 0+0=0,
$$

where $\gamma_{k} \in \mathbb{D}$. Therefore $x$ is a $\mathbb{D}$-supercyclic vector for $B_{\omega}$.

To complete our work, we must consider that the zero in the boundary of $\Gamma$ and exhibit an example of a $\mathbb{D}$-supercyclic operator that is not $\Gamma$-supercyclic.

Example 3.3. Let $\Gamma=\left\{\frac{1}{n}: n \in \mathbb{N}\right\}$ be a bounded subset of $\mathbb{C}$. Let $T: \mathbb{C} \longrightarrow \mathbb{C}$ be defined by $T(z)=2 z$ for all $z \in \mathbb{C}$. Then $T$ is $\mathbb{D}$-supercyclic but not $\Gamma$-supercyclic.

Proof. It is not so hard to notice that 0 is in the boundary of $\Gamma$. By [1] $T$ is $\mathbb{D}$-supercyclic. On the other hand we claim that there are no $\Gamma$-supercyclic vectors in $\mathbb{C}$. In order to show that, take $y$ be a non-zero vector in $\mathbb{C}$, and consider $\operatorname{\Gamma rb}(T, y)$ be the scaled orbit of $y$ with $T$. We show that $\operatorname{\Gamma Orb}(T, y)$ is not dense in $\mathbb{C}$. For instance, take the vector $i y$ and choose $\varepsilon=\frac{\|y\|}{2}$. One can show that $\left\|\frac{1}{n} T^{m} y-i y\right\|=\left\|\frac{1}{n} 2^{m} y-i y\right\|=\left|\frac{1}{n} 2^{m}-i\right|\|y\|=\left(\sqrt{\frac{2^{2 m}}{n^{2}}+1}\right)\|y\|>\|y\|$, for any $n, m \in \mathbb{N}$. Hence $B(i y, \varepsilon) \cap \operatorname{\Gamma orb}(T, y)=\emptyset$.

Now, it is time to present the following Theorem.

Theorem 3.4. Let $\Gamma \backslash\{0\}$ be a non-empty subset of $\mathbb{C}$. If $\Gamma$ is a diskcyclic scaler set then $\Gamma$ is bounded and $0 \in \Gamma^{\circ}$.

Proof. Suppose that $\Gamma$ is unbounded or $0 \in / \bar{\Gamma}$. By using Definition 2.2 and above three examples, we get the desire result. $\mathbf{D}$ Now, thanks to Theorem 3.4, the necessity part of Theorem A is proved. 


\section{SUFFICIENTLY PART of THEOREM A}

Now, we will complete the second direction of Theorem A, that is, if $\Gamma$ is bounded and $0 \in \Gamma^{\circ}$ then $\Gamma$ is a diskcyclic scalar set "then $T$ is diskcyclic". Indeed, this direction give the sufficient part of theorem A.

Proposition 4.1. Let $\Gamma \subset \mathbb{C}$ such that $\Gamma \backslash\{0\}$ is non-empty, bounded and $0 \in \Gamma^{\circ}$. Then $\Gamma$ is a diskcyclic scalar set.

Proof. Suppose that $\Gamma$ is a bounded subset of $\mathbb{C}$ and $0 \in \Gamma^{\circ}$. To prove that $\Gamma$ is a diskcyclic scaler set it suffices to show that for any separable complex Banach space $X$ and for any $T \in \mathcal{B}(X), T$ is $\Gamma$-supercyclic if and only if $T$ is $\mathbb{D}$-supercyclic. Firstly, suppose that $T$ is $\Gamma$-supercyclic on a separable complex Banach space $X$. Let $\varepsilon>0$ be given. Since $\Gamma$ is bounded there exists $c>0$ such that for any $\gamma \in \Gamma,|\gamma| \leq c$. By the $\Gamma$-supercyclicity of $T$ we have for any non-zero vector $y \in X$, there are $\alpha \in \Gamma$ and $n \in \mathbb{N}$ such that $\left\|\alpha T^{n} x-c y\right\|<c \varepsilon$. Hence, $\left\|\frac{\alpha}{c} T^{n} x-y\right\|<\varepsilon$. we notice that $\left|\frac{\alpha}{c}\right| \leq 1$. Hence, $T$ is a $\mathbb{D}$-supercyclic operator.

Conversely, suppose that $T$ is a $\mathbb{D}$-supercyclic operator on a separable complex Banach space $X$. Since $0 \in \Gamma^{\circ}$ there is $\varepsilon>0$ such that $B(0, \varepsilon)$ is included in $\Gamma$. In other word, there is a disk of radius $\varepsilon$ about the zero in $\Gamma$. Thus, by Proposition (2.1) we have $T$ is a $\mathbb{D}_{\varepsilon}$-supercyclic. Since $\mathbb{D}_{\varepsilon} \subset \Gamma$, it is obvious that $T$ is $\Gamma$-supercyclic on $X$ with the same vector.

After using Theorem (3.4) and Proposition (4.1) we have already given a complete proof of Theorem A and consequentially a positive answer of Question 1. Roughly speaking we can say that, for any operator $T$ on a separable complex Banach space, $T$ is diskcyclic if and only if $T$ is $\Gamma$-supercyclic where $\Gamma$ is bounded and $0 \in \Gamma^{\circ}$.

In the following, we prove that the density of the orbit of diskcyclic vectors in Hilbert space is equivalent with the density of the image of the orbit under a bounded linear functional in complex plane.

Proposition 4.2. [6] Let $\mathcal{H}$ be a Hilbert space. Let $z$ be a fixed non-zero element in $\mathcal{H}$. Define $f_{z}: \mathcal{H} \rightarrow \mathbb{C}$ by $f_{z}(y):=<y, z>$ for every $y \in \mathcal{H}$. Then $f_{z}$ is an injective bounded linear functional, such that $\left\|f_{z}\right\|=\|z\|$ for every $z \in \mathcal{H}$.

Theorem 4.3. Let $\Gamma \subseteq \mathbb{C}$ and $T \in \mathcal{B}(\mathcal{H})$ be a $\Gamma$-supercyclic operator with a $\Gamma$-supercyclic vector $x$. The $\operatorname{Orb}(\Gamma x, T)$ is dense in $\mathcal{H}$ if and only if $f_{x}(\operatorname{Orb}(\Gamma x, T))=\left\{f_{x}\left(T^{n} \gamma x\right)=<T^{n} \gamma x, x>: \gamma \in\right.$ $\Gamma, n=0,1,2, \ldots\}$ is dense in $\mathbb{C}$.

Proof.

Assume that $\operatorname{Orb}(\Gamma x, T)=\left\{T^{n} \gamma x: \gamma \in \Gamma, n=0,1,2, \ldots\right\}$ is dense in $\mathcal{H}$. Let $\alpha$ be an arbitrary element in $\mathbb{C}$. Take $\langle\alpha, x\rangle=\lambda_{0}$ for some $\lambda_{0} \in \mathbb{C}$. Now, there exist $\zeta \in \mathbb{C}$ such that $\lambda=\zeta \lambda_{0}$, which implies, $\lambda=\zeta<\alpha, x>=<\zeta \alpha, x>=f_{x}(\alpha \zeta)$. By the density of the $\operatorname{Orb}(\Gamma x, T)$ there exist a sequence $\left(n_{j}\right) \subset \mathbb{N}$ and $\gamma_{0} \in \Gamma$ such that $T^{n_{j}} \gamma_{0} x \rightarrow \alpha \zeta$. Since $f_{x}$ is bounded linear functional then $f_{x}\left(T^{n_{j}} \gamma_{0} x\right) \rightarrow f_{x}(\alpha \zeta)$ or $<T^{n_{j}} \gamma_{0} x, x>\rightarrow<\alpha \eta, x>=\lambda$. Hence $\left\{f_{x}\left(T^{n} \gamma x\right)=<T^{n} \gamma x, x>: \gamma \in\right.$ 
$\Gamma, n=0,1,2, \ldots\}$ is dense in $\mathbb{C}$.

Conversely, suppose that $\left\{f_{x}\left(T^{n} \gamma x\right)=<T^{n} \gamma x, x>: \gamma \in \Gamma, n=0,1,2, \ldots\right\}$ is dense in $\mathbb{C}$. Let

$y \in H$ be arbitrary, then $f_{x}(y)=<y, x>\in \mathbb{C}$. By the density of the later set there exist a sequence $\left(n_{j}\right) \subset \mathbb{N}$ and $\gamma_{0} \in \mathbb{D}$ such that $<T^{n_{j}} \gamma_{0} x, x>\rightarrow<y, x>$. Now $\left\|T^{n_{j}} \gamma_{0} x-y\right\|=\| T^{n_{j}} \gamma_{0} x-f_{x}^{-1}(<$ $y, x>)\|=\| f_{x}^{-1}\left[f_{x}\left(T^{n_{j}} \gamma_{0} x\right)-<y, x>\right]\|\leq\| f_{x}^{-1}\|\| f_{x}\left(T^{n_{j}} \gamma_{0} x\right)-<y, x>\| \rightarrow 0$ because $f_{x}^{-1}$ is bounded. Thus $T^{n_{j}} \gamma_{0} x \rightarrow y$ in $H$. The inverse map was used above because the element $\langle y, x>$ was in the range of $f_{x}$. Therefore, $\operatorname{Orb}(\Gamma x, T)=\left\{T^{n} \gamma x: \gamma \in \mathbb{D}, n=0,1,2, \ldots\right\}$ is dense in $\mathcal{H}$.

We consider that the previous result is useful to recognize the $\mathbb{D}$-supercyclic operators, because one can discuss the diskcyclicity of any operator on Hilbert space by check the density of the set $\left\{<T^{n} \gamma x, x>: \gamma \in \mathbb{D}, n=0,1,2, \ldots\right\}$ in $\mathbb{C}$ for such $\mathbb{D}$-supercyclic vector $x$.

\section{Conclusion}

In this article we introduce a complete characterization of the subset $\Gamma \subset \mathbb{C}$ which has an equivalence to the closed unit disk $\mathbb{D} \subset \mathbb{C}$. In addition, we present a new idea to how construct $\mathbb{D}$-supercyclic operators and the corresponding $\mathbb{D}$-supercyclic vector on $\ell^{2}$ spaces. In light of these result, we will deal with flexibility with $\mathbb{D}$-supercyclic operators and hope to get new results in further researches.

\section{References}

[1] N. Bamerni, A. Kilicman, and M. Noorani. A review of some works in the theory of diskcyclic operators. Bulletin of the Malaysian Mathematical Sciences Society. Journal of Functional Analysis, Elsevier, pages 1-17, 2015.

[2] G. D. Birkhoff. Surface transformations and their dynamical applications. Acta Math., 43:1-119, 1922.

[3] G. D. Birkhoff. Demonstration d'un theoreme elementaire sur les fonctions entieres. C. R. Acad. Sci. Paris, 189:473-475, 1929.

[4] S. Charpentier and R. Ernst. Hypercyclic subsets. Journal, 140:759-798, 2020.

[5] S. Charpentier, R. Ernst, and Q. Menet. Г-supercyclicity. Journal of Functional Analysis, Elsevier, 270(12):4443-4465, 2016.

[6] F. Deutsch. Best Approximation in Inner Product Spaces., volume 7 of CMS Books in Mathematics. Springer, New York, 2001.

[7] N. Feldman, V. Müller, and L. Müller. Hypercyclic and supercyclic cohyponormal operators. Acta Sci. Math., 68:303-328, 2002.

[8] KG. Grosse-Erdmann and A. Peris. Linear Chaos. Universitext. Springer, London, 2011. 
[9] D.A. Herrero. Hypercyclic operators and chaos. J. Operator Theory, 28:93-103, 1992.

[10] H. M. Hilden and L.J. Wallen. Some cyclic and non-cyclic vectors of certain operators. Indiana Univ. Math. J., 23:557-565, 1974.

[11] Z. Jamil. Cyclic Phenomena of Operators on Hilbert Space. PhD thesis, University of Bahdad, Iraq, 2002.

[12] F. Leon-Saavedra and V. Müller. Hypercyclic sequences of operators. Studia Math., 175:1-18, 2006.

[13] G. MacLane. Sequences of derivatives and normal families. J. Analyse Math., 1952. 2:72-87. 